\title{
artigo
}

Menezes, C.A.; Costa, G.L.O.B.; Barreto, R.F.; Oliveira, V.S.;

Proteína C reativa importante biomarcador de risco cardiometabólico na obesidade infanto-juvenil

\section{Proteína C reativa importante biomarcador de risco cardiometabólico na obesidade infanto-juvenil}

\author{
C-reactive protein important biomarker of cardiometabolic risk in childhood obesity \\ Proteína C reactiva importante biomarcador de riesgo cardiometabólico en la obesidad infantil
}

\begin{abstract}
RESUMO
Estabelecer a importância da avaliação da proteína C reativa ultrassensivel (PCR-us) como biomarcador em um grupo pediátrico obeso, detectando precocemente possiveis complicações cardiometabólicas. Trata-se de estudo caso-controle envolvendo 342 crianças e adolescentes, do Serviço de Medicina Preventiva, Aracaju-Sergipe, Brasil. Participaram do estudo 235 obesos e 107 controles. A PCR-us apresentou valor médio de $2,36 \pm 1,28 \mathrm{mg} / \mathrm{dL}$ no grupo obeso e $0,01 \pm 0,1 \mathrm{mg} / \mathrm{dL}$ no grupo controle. Observou-se correlação significativa do aumento de PCR-us no grupo obeso com achados bioquímicos e antropométricos como: redução do HDL, elevação de triglicérides e com os maiores indicadores de índice de massa corporal e da circunferência abdominal. A homocisteína demonstrou ser um biomarcador pouco especifico neste estudo. Portanto, a proteína $C$ reativa ultrassensivel demonstrou ser um biomarcador de risco cardiometabólico, apresentando alta sensibilidade em nossa população pediátrica com obesidade.
\end{abstract}

DESCRITORES: Proteína C-Reativa; Obesidade; Pediatria.

\section{ABSTRACT}

To establish the importance of evaluating ultrasensitive C-reactive protein (hs-CRP) as a biomarker in an obese pediatric group, detecting possible cardiometabolic complications early. This is a case-control study involving 342 children and adolescents, from the Preventive Medicine Service, Aracaju-Sergipe, Brazil. 235 obese and 107 controls participated in the study. The CRP-us showed a mean value of $2.36 \pm 1.28 \mathrm{mg} / \mathrm{dL}$ in the obese group and $0.01 \pm 0.1 \mathrm{mg} / \mathrm{dL}$ in the control group. There was a significant correlation between the increase in hs-CRP in the obese group and biochemical and anthropometric findings such as: reduced HDL, elevated triglycerides and with the highest indicators of body mass index and abdominal circumference. Homocysteine proved to be a poorly specific biomarker in this study. Therefore, ultrasensitive C-reactive protein has been shown to be a biomarker of cardiometabolic risk, presenting high sensitivity in our pediatric population with obesity.

DESCRIPTORS: C-Reactive Protein; Obesity; Pediatrics.

\section{RESUMEN}

Establecer la importancia de evaluar la proteína C reactiva ultrasensible (hs-PCR) como biomarcador en un grupo pediátrico obeso, detectando precozmente posibles complicaciones cardiometabólicas. Se trata de un estudio de casos y controles que involucró a 342 niños y adolescentes, del Servicio de Medicina Preventiva, Aracaju-Sergipe, Brasil. 235 obesos y 107 controles participaron en el estudio. El CRP-us mostró un valor medio de 2,36 $\pm 1,28 \mathrm{mg} / \mathrm{dL}$ en el grupo de obesos y 0,01 $\pm 0,1 \mathrm{mg} / \mathrm{dL}$ en el grupo control. Hubo una correlación significativa entre el aumento de hs-PCR en el grupo de obesos y hallazgos bioquímicos y antropométricos como: HDL reducido, triglicéridos elevados y con los mayores indicadores de índice de masa corporal y circunferencia abdominal. La homocisteína demostró ser un biomarcador poco específico en este estudio. Por tanto, la proteína $C$ reactiva ultrasensible ha demostrado ser un biomarcador de riesgo cardiometabólico, presentando una alta sensibilidad en nuestra población pediátrica con obesidad.

DESCRIPTORES: Proteína C-Reactiva; Obesidad; Pediatría.

RECEBIDO EM: 27/01/2021 APROVADO EM: 11/02/2021

\section{Carlos Alberto Menezes}

Professor Adjunto de Endocrinologia e Metabologia do curso de Medicina e docente do Programa de Pós-Graduação em Ciências da Saúde da Universidade Estadual de Santa Cruz (PPGCS/UESC). Médico Endocrinologista do Centro de Especialidades Médicas da Criança e Adolescentes da cidade de Aracaju-Sergipe (CEMCA).

ORCID: 0000-0003-2306-6494 


\section{Giovanna Lúcia Oliveira Bonina Costa}

Nutricionista. Mestre em Alimentos, Nutrição e Saúde - Programa de Pós-Graduação em Alimentos, Nutrição e Saúde da Universidade Federal da Bahia (PGNUT/UFBA). Discente do curso de Medicina da Universidade Estadual de Santa Cruz (UESC). ORCID: 0000-0002-4208-4746

\section{Rafael Ferreira Barreto}

Discente do curso de Medicina da Universidade Estadual de Santa Cruz (UESC). ORCID: 0000-0003-3261-1108

\section{Victória Santos Oliveira}

Discente do curso de Medicina da Universidade Estadual de Santa Cruz (UESC). ORCID: 0000-0002-4275-2273

\section{INTRODUÇÃO}

A obesidade infantil é considerada uma epidemia de crescimento mundial, devido a forte influência ambiental, comportamental e genética, os quais contribuem para o acúmulo de gordura e portanto, na composição corpo$\mathrm{ral}^{1,2}$. Esse crescimento acentuado da obesidade nesse período pode ser responsável pelo aparecimento precoce de distúrbios cardiometabólicos ${ }^{2}$.

A tendência mundial acerca do incremento no índice de massa corporal (IMC) demonstrou na fase infantil um aumento global desse indicador por década de $0,32 \mathrm{~kg} / \mathrm{m}^{2}$ nas meninas e $0,40 \mathrm{Kg} / \mathrm{m}^{2}$ nos meninos. Isso resultou em um aumento da obesidade infantil de $0,7 \%$ para $5,6 \%$ e de $0,9 \%$ para $7,8 \%$ nos sexos feminino e masculino respectivamente nesse período3. No entanto, nota-se uma estabilização do crescimento da obesidade infantil em países desenvolvidos e sua manutenção em países em desenvolvimento ${ }^{4,5}$. No Brasil, a Pesquisa de Orçamentos Familiares (POF), em parceria com o Ministério da Saúde (MS), demonstrou que $33,5 \%$ das crianças entre 5-9 anos estavam acima do peso, sendo que $16,6 \%$ dos meninos e $11,8 \%$ das meninas eram obesos; já na faixa etária de 10-19 anos, o excesso de peso foi de $21,7 \%$ no sexo masculino e $19,4 \%$ no sexo feminino, sendo a região sudeste correspondente à área com maior apresentação de sobrepeso infantil ${ }^{6,7}$.

A obesidade é considerada uma doença crônica complexa e multifatorial, resultante de um longo período de balanço energético positivo, no qual fatores genéticos e ambientais estão envolvidos, bem como a associação de um processo inflamatório de baixo grau ${ }^{8,9}$. Atualmente, há indícios de que o tecido adiposo não tenha apenas a função de reservatório energético, mas também atue como um órgão endócrino ativo, que age de diversas formas nos sistemas orgânicos, contribuindo com o processo inflamatório em indivíduos obesos. Os valores anormais de metabólitos como os lipídeos, ácidos graxos e citocinas liberados pelo tecido adiposo atuam ativando mocrófagos $\mathrm{M} 1$ e aumentando a resposta inflamatória, por meio do aumento da secreção de citocinas, as quais estimulam macrófagos e adipócitos a produzir adipocinas pró-inflamatórias, como o fator de necrose tumoral alfa (TNF-alfa), interleucina-1(IL-1), interleucina-6 (IL-6) e substâncias pró-coagulantes10,11. Dentre essas, um destaque se dá a IL-6 que estimula o fígado a produzir proteína $\mathrm{C}$ reativa (PCR). Por outro lado, o tecido adiposo subcutâneo produz a adiponectina, hormônio que de forma antagônica às demais adipocinas eleva a sensibilidade à insulina nos tecidos e atua como hormônio protéico anti-inflamatório, anti-adipogênico e anti-aterogênico ${ }^{10}$.

Várias condições clínicas, como infecção, trauma e inflamação, resultam em alterações sistêmicas diretas e indiretas significativas culminado com a elevação da proteína $C$ reativa (PCR). Nesse processo, tem-se uma regulação da produção de óxido nítrico (NO), aumento de lipoproteína de baixa densidade (LDL-c), facilitação da ação de genes pró ateroscleróticos, inibição da adiponectina, vasoconstrição e estímulo à adesão e ativação plaquetária, podendo ocasionar oclusão e formação de trombos. Dessa forma, a PCR, além de um marcador inflamatório de aterosclerose e eventos coronarianos, é também um mediador de doença devido à sua contribuição na formação de lesão $0^{8,10,12}$.

Uma vez determinado o papel da inflamação no desenvolvimento da obesidade e da doença aterosclerótica, diferentes biomarcadores inflamatórios foram pesquisados e identificados com o intuito de adicionar valor prognóstico ao risco cardiovascular mesmo no grupo pediátrico. Entretanto, a literatura mundial não é unânime no que diz respeito à correlação entre a PCR ultrassensível (PCR-us) e o risco cardiometabólico na obesidade infanto-juvenil ${ }^{13}$.

Portanto, este estudo objetivou estabelecer a importância da avaliação da PCR-us em nosso grupo pediátrico com obesidade e demonstrar a sua importância como um biomarcador, cujo intuito é detectar precocemente possíveis complicações cardiometabólicas nessa faixa etária.

\section{MATERIAL E MÉTODOS}

\section{Amostra}

Trata-se de um estudo caso-controle com uma amostra de 342 indivíduos com idade entre 6 e 18 anos, sendo 235 crianças e adolescentes obesos (128 do sexo masculino e 107 do sexo feminino) e 107 do grupo de controle, composto por crianças e adolescentes controles ( 52 do sexo masculino e 55 do sexo feminino). Devido à heterogeneidade da amostra, dividimos os indivíduos em três faixas etárias de acordo com a Federação Internacional de Diabe- 
tes (FID): 6-10 anos, 10-16 anos e maior que 16 anos.

O estudo foi desenvolvido no Serviço de Medicina Preventiva (SEMPRE) em Aracaju-Sergipe, Brasil. O trabalho foi aprovado pelo Comitê de Ética em Pesquisa da Universidade Estadual de Santa Cruz (UESC), via Plataforma Brasil e informado o consentimento do estudo sobre o número 04065412.600005526 .

\section{Avaliações antropométrica e bioquímica}

Inicialmente, foram avaliados os seguintes parâmetros antropométricos dos indivíduos: peso, altura, IMC, circunferência abdominal (CA) e pressão arterial (PA). Para a verificação do peso, utilizou-se a balança antropométrica (Filizola Brasil). A caracterização da obesidade se baseou nas tabelas de IMC da Organização Mundial da Saúde (OMS), seguindo a comparação gênero e idade30. Os indivíduos foram considerados obesos quando o valor do IMC se encontrava acima do percentil 97 ou o cálculo do Z- escore do IMC for maior que +2 . A circunferência abdominal foi medida no ponto médio entre o rebordo costal inferior e a maior elevação da crista ilíaca e a pressão arterial foi mensurada utilizando manguitos com circunferência e largura apropriados para a faixa etária referida.

Posteriormente, os grupos foram avaliados bioquimicamente através das dosagens da GJ (glicemia de jejum), insulina, Homeostasis model assesmentof insulin resistence (HOMA-IR), AST (aspartato amino transferase), ALT (alanina amino transferase), gama GT (gama glutamil transferase), ácido úrico, colesterol total (CT), lipoproteína de baixa densidade (LDL-c), lipoproteína de alta densidade (HDL), triglicérides (TG), hemograma completo, TSH (hormônio tireoestimulante), T4 livre (tiroxina livre), IGF-1 (fator de crescimento semelhante à insulina), cortisol urinário, além dos biomarcadores homocisteína e PCR-us.

\section{Mensuração laboratorial de biomar- cadores de risco cardiometabólico}

A PCR-us foi dosada pelo método de imunoturbidimetria utilizando o aparelho Immage (BeckmanCoulter, USA). Os valores utilizados para a estratificação do risco cardiovascular (RCV) foram: RCV baixo: < 0,1 mg/dL, RCV médio: 0,1 $0,3 \mathrm{mg} / \mathrm{dL}$ e RCV alto: $>0,3 \mathrm{mg} / \mathrm{dL}$.

A homocisteína total plasmática foi determinada pelo método de quimioluminescência, com valor de referência

\begin{tabular}{|c|c|c|c|c|}
\hline \multirow{3}{*}{ IMC (Kg/m2) } & & 6A 10 ANOS & 10 A 16 ANOS & $>16$ ANOS \\
\hline & Controle & $17,74 \pm 0,99$ & $19,92 \pm 0,60$ & $18,75 \pm 0,19$ \\
\hline & Obeso & $26,21 \pm 0,49^{* * *}$ & $29,11 \pm 0,59^{* * *}$ & $33,90 \pm 1,37^{* *}$ \\
\hline \multirow{2}{*}{$\mathrm{CA}(\mathrm{cm})$} & Controle & $64,39 \pm 1,28$ & $67,30 \pm 0,72$ & $70,5 \pm 0,5$ \\
\hline & Obeso & $84,00 \pm 1,17^{* * *}$ & $96,07 \pm 1,83^{* * *}$ & $105,8 \pm 3,92^{* *}$ \\
\hline \multirow{2}{*}{ GJ (mg/dL) } & Controle & $85,78 \pm 1,57$ & $84,90 \pm 1,18$ & $88,25 \pm 2,75$ \\
\hline & Obeso & $86,92 \pm 1,23$ & $87,61 \pm 1,25$ & $99,90 \pm 1,18^{*}$ \\
\hline \multirow{2}{*}{$\mathrm{CT}$ (mg/dL) } & Controle & $157,0 \pm 5,13$ & $156,7 \pm 5,56$ & $126,8 \pm 16,2$ \\
\hline & Obeso & $180,1 \pm 4,29 * *$ & $175,4 \pm 3,45^{* *}$ & $211,3 \pm 8,59^{* *}$ \\
\hline \multirow{2}{*}{ LDL (mg/dL) } & Controle & $92,72 \pm 4,55$ & $102,4 \pm 7,10$ & $93,75 \pm 6,25$ \\
\hline & Obeso & $109,1 \pm 4,31^{* * *}$ & $110,8 \pm 4,04^{* * *}$ & $138,8 \pm 14,3^{* *}$ \\
\hline \multirow{2}{*}{$\mathrm{HDL}$ (mg/dL) } & Controle & $49,61 \pm 1,81$ & $45,30 \pm 1,85$ & $47,50 \pm 2,5$ \\
\hline & Obeso & $39,02 \pm 1,22^{* * *}$ & $37,98 \pm 1,15^{* * *}$ & $37,10 \pm 0,86^{* *}$ \\
\hline
\end{tabular}

menor que $15 \mathrm{mcmol} / \mathrm{L}$. A insulina plasmática também foi determinada pelo método quimioluminescência, sendo o valor de referência inferior a $28,4 \mathrm{mU} / \mathrm{mL}$. O HOMA-IR foi calculado multiplicando a insulina de jejum $(\mathrm{mU} / \mathrm{mL})$ x glicemia de jejum $(\mathrm{mg} / \mathrm{dL}) / 405$. O valor de corte utilizado para caracterização da resistência insulínica foi maior ou igual a 3,16.

\section{Análise estatística}

Os resultados foram analisados estatisticamente pelo teste de Mann Whitney, adotando-se um $\mathrm{p}<0,05$ como nível de significância. Os resultados foram obtidos através da média, do erro padrão da média e intervalo de confiança. Para análise estatística foi usado o software GraphPad Prisma versão 5.1.

\section{RESULTADOS}

Foram avaliadas 342 crianças e adolescentes, sendo 235 do grupo de obesos (128 do sexo masculino e 107 do sexo feminino), e 107 indivíduos do grupo controle não-obeso ( 52 do sexo masculino e 55 do sexo feminino). O intervalo médio das idades cronológicas foi de $10 \pm 2,3$ anos para ambos os grupos.

Os dados antropométricos e bioquímicos do grupo obeso do sexo masculino em comparação ao controle conforme a faixa etária estão expostos na Tabela 1. Observa-se que, em todas as faixas etárias avaliadas, os indivíduos obesos apresentaram alteração significativa dos parâmetros antropométricos, com elevação do IMC e aumento da CA em comparação ao grupo controle $(6$ a 16 anos $\mathrm{p}<0,0001>16$ anos $\mathrm{p}<0,001)$. Além disso, verificou-se que esses indivíduos apresentaram aumento significativo do CT $(\mathrm{p}<0,001) \mathrm{em}$ todas as faixas etárias, aumento do LDL-c, TG e redução do HDL-c, sendo estas diferenças mais proeminentes na faixa etária até 16 anos $(p<0,001)$. Em relação ao perfil glicêmico, os indivíduos com idade superior a 16 anos apresentaram glicemia de jejum acima do grupo controle $(\mathrm{p}<0.05)$. Os resultados também revelaram que a PCR-us estava aumentada em todos os grupos avalia$\operatorname{dos}(\mathrm{p}<0,0001)$. 


$\begin{array}{lcccc}\text { TG }(\mathrm{mg} / \mathrm{dL}) & \text { Controle } & 77,11 \pm 6,72 & 90,40 \pm 10,5 & 71,00 \pm 6,0 \\ & \text { Obeso } & 147,0 \pm 1,96^{* * *} & 136,7 \pm 7,7^{* * *} & 235,5 \pm 27,5^{* *} \\ \text { PCR-us (mg/dL) } & \text { Controle } & 0,06 \pm 0,01 & 0,06 \pm 0,01 & 0,01 \pm 0,01 \\ & \text { Obeso } & 1,43 \pm 0,12^{* * *} & 2,33 \pm 0,17^{* * *} & 2,82 \pm 0,35^{* * *}\end{array}$

Fonte: Autores do estudo

IMC (índice de massa corpórea), CA (circunferência abdominal), GJ (glicemia de jejum), CT (colesterol total), LDL-c (lipoproteína de baixa densidade), HDL (lipoproteína de alta densidade), TG (triglicérides). * $\mathrm{p}<0,05,{ }^{* *} \mathrm{p}<0,001 \mathrm{e}^{* * *} \mathrm{p}<0,0001$ em comparação ao grupo controle.

TABELA 2: Dados antropométricos e bioquímicos em um grupo de crianças e adolescentes do sexo feminino, controle $(n=55)$ e com obesidade $(n=107)$.

$\begin{array}{lcccc} & & \text { 6A 10 ANOS } & \text { 10 A 16 ANOS } & >16 \text { ANOS } \\ \text { IMC (Kg/m2) } & \text { Controle } & 17,99 \pm 0,84 & 17,91 \pm 0,41 & 22,5 \pm 0,1 \\ & \text { Obeso } & 25,39 \pm 0,45^{* * *} & 29,11 \pm 0,71^{* * *} & 30,40 \pm 1,13^{*} \\ \text { CA (cm) } & \text { Controle } & 62,27 \pm 2,01 & 65,75 \pm 1,01 & 70,0 \pm 0,1 \\ & \text { Obeso } & 82,97 \pm 1,29^{* * *} & 90,02 \pm 1,33^{* * *} & 96,50 \pm 3,17^{*} \\ \text { GJ (mg/dL) } & \text { Controle } & 83,93 \pm 2,07 & 85,67 \pm 1,54 & 88,5 \pm 0,1 \\ & \text { Obeso } & 84,74 \pm 1,35 & 87,38 \pm 1,03 & 96,00 \pm 2,04^{*} \\ \text { CT (mg/dL) } & \text { Controle } & 128,1 \pm 4,37 & 141,5 \pm 3,80 & 120,2 \pm 0,1 \\ & \text { Obeso } & 172,7 \pm 4,35^{* * *} & 168,8 \pm 4,45^{* *} & 182,3 \pm 4,23 \\ \text { LDL (mg/dL) } & \text { Controle } & 99,73 \pm 7,11 & 96,00 \pm 3,52 & 99,0 \pm 0,1 \\ & \text { Obeso } & 110,7 \pm 4,04^{*} & 104,1 \pm 3,68 & 107,3 \pm 5,4 \\ \text { HDL (mg/dL) } & \text { Controle } & 58,87 \pm 2,33 & 45,76 \pm 1,41 & 48,0 \pm 0,1 \\ & \text { Obeso } & 39,0 \pm 0,96^{* * *} & 38,2 \pm 1,20^{*} & 39,75 \pm 0,5 \\ \text { TG (mg/dL) } & \text { Controle } & 74,93 \pm 4,04 & 81,95 \pm 3,21 & 110 \pm 2,0 \\ & \text { Obeso } & 152,4 \pm 8,67^{* * *} & 158,9 \pm 8,8^{* * *} & 159,0 \pm 7,4 \\ \text { PCR-us (mg/dL) } & \text { Controle } & 0,039 \pm 0,009 & 0,04 \pm 0,009 & 0,02 \pm 0,1 \\ & \text { Obeso } & 1,85 \pm 0,15^{* * *} & 2,27 \pm 0,37^{* * *} & 2,92 \pm 0,14 *\end{array}$

Fonte: Autores do estudo

IMC (índice de massa corpórea), CA (circunferência abdominal), GJ (glicemia de jejum), CT (colesterol total), LDL-c (lipoproteína de baixa densidade), HDL (lipoproteína de alta densidade), TG (triglicérides). ${ }^{*} \mathrm{p}<0,05,{ }^{* *} \mathrm{p}<0,001 \mathrm{e}{ }^{* * *} \mathrm{p}<0,0001$ em comparação ao grupo controle.

TABELA 3: Dados bioquímicos e hormonais de um grupo de crianças e adolescentes de ambos os sexos, controle $(n=107)$ e com obesidade $(n=235)$.
TESTES

Leucócitos (mm3)

PCR- us (mg/dL)

Ácido Úrico (mg/dL)

Insulina (UI/ml)

HOMA-IR

$\mathrm{CT}(\mathrm{mg} / \mathrm{dL})$

$\mathrm{HDL}$ (mg/dL)

$\operatorname{LDL}(\mathrm{mg} / \mathrm{dL})$

TG (mg/dL)

AST (U/L)

ALT (U/L)

\section{CONTROLE}

$6900 \pm 600$

$0,01 \pm 0,1$

$4,6 \pm 1,05$

$14,9 \pm 6,2$

2,0

$170 \pm 36$

$44 \pm 9,3$

$94 \pm 19$

$111 \pm 60$

$21 \pm 2,07$

$23 \pm 1,6$
OBESO

$7600 \pm 450$

$2,36 \pm 1,28^{* * *}$

$5,85 \pm 1,14^{* * *}$

$18,9 \pm 5,4^{* * *}$

$3,96^{* * *}$

$237 \pm 34^{* * *}$

$38 \pm 7,9^{* * *}$

$154 \pm 39^{* *}$

$180 \pm 13^{* * *}$

$32 \pm 2,7^{* * *}$

$37 \pm 1,3^{* * *}$
As variáveis antropométricas e bioquímicas dos grupos com obesidade do sexo feminino em comparação ao controle conforme a faixa etária estão expostas na Tabela 2. Nota-se que os parâmetros antropométricos IMC e CA estavam significativamente elevados nos grupos de crianças com obesidade em relação ao grupo controle, com diferenças maiores nas faixas etárias até 16 anos $(\mathrm{p}<0,0001)$. Com relação ao perfil lipídico, o CT do grupo obeso estava elevado nos indivíduos de 6 a 10 anos $(p<0,0001)$ e 10 e 16 anos $(\mathrm{p}<0,001)$ e o LDL aumentado somente no grupo obeso de 6 a 10 anos $(p<0,05)$. O HDL se apresentou reduzido no grupo com obesidade nas faixas etárias de 6 a $10(\mathrm{p}<0,0001)$ e 10 a 16 anos $(\mathrm{p}<0,05)$, os triglicérides se mostraram elevados nos indivíduos obesas menores de 16 anos $(\mathrm{P}<0,0001)$. Semelhante ao sexo masculino, níveis aumentados da glicemia só foram observados nos indivíduos obesos maiores de 16 anos com $\mathrm{p}<0,05$. A PCR-us encontrou-se elevada em todas as faixas etárias do grupo obeso em relação ao controle, com maiores diferenças nos menores de 16 anos $(\mathrm{p}<0,0001)$.

Os resultados referentes à avaliação laboratorial do grupo obeso em relação ao controle de toda a amostra do estudo estão explicitados na Tabela 3. O grupo com obesidade apresentou alteração das variáveis bioquímicas analisadas como ácido úrico, insulina, HOMA-IR, CT, HDL, LDL, TG, AST, ALT, GGT. Por outro lado, não foi verificada diferença na dosagem hormonal entre os grupos obeso e controle, além do leucograma não ter apresentado diferença significativa nos grupos avaliados.

Os níveis de PCR-us e homocisteína no grupo obeso e controle, em função da faixa etária e sexo, estão evidenciadas na Figura 1. Os resultados revelaram que o grupo obeso apresenta níveis mais elevados de PCR-us, em todas as faixas etárias e em ambos os sexos quando comparado ao grupo controle. Tais diferenças estatísticas foram bastante significativas no sexo masculino na faixa etária inferior a 16 anos $(p<0,0001)$. Não foi possível detectar di- 


$\begin{array}{ccc}\text { GGT (U/L) } & 29 \pm 12 & 54 \pm 9,2^{* * *} \\ \text { TSH (mUl/ml) } & 1,10 \pm 1,19 & 2,07 \pm 1,16 \\ \text { T4 livre (ng/dL) } & 1,07 \pm 0,2 & 1,03 \pm 0,29 \\ \text { Cortisol urinário (ug/dL) } & 9,78 \pm 4,75 & 9,38 \pm 4,88 \\ \text { IGF-1 (ng/ml) } & 231 \pm 83 & 245 \pm 111\end{array}$

Fonte: Autores do estudo

GJ (glicemia de jejum), HOMA-IR (Homeostasismodelassesmentofinsulinresistence), CT (colesterol total), HDL (lipoproteína de alta densidade), LDL-c (lipoproteína de baixa densidade), TG (triglicérides). AST (aspartatoaminotransferase), ALT (alaninaaminotransferase), GGT (gama glutamil transferase), TSH (hormôniotireoestimuante), T4 livre (tiroxina livre), IGF-1 (fator de crescimento semelhante à insulina), PCR-us (proteína C reativa ultrassensivel). ${ }^{*} p<0,05,{ }^{* *} p<0,001$ e ${ }^{* * *} p<0,0001$ em comparação ao grupo controle.

Figura 1. Representação do comportamento da proteína C reativa (PCR-us) e homocisteína em relação aos grupos obeso e controle do sexo masculino ( $\mathrm{A}$ e C) e feminino (B e D) nas faixas etárias estudadas. ${ }^{*} p<0,05,{ }^{* *} p<0,01,{ }^{* * *} p<0,0001$.
A
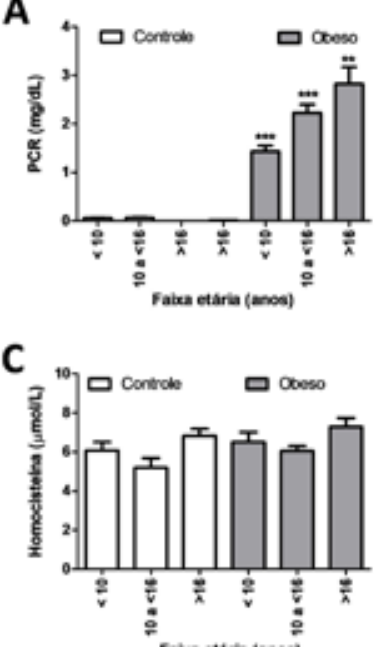

B

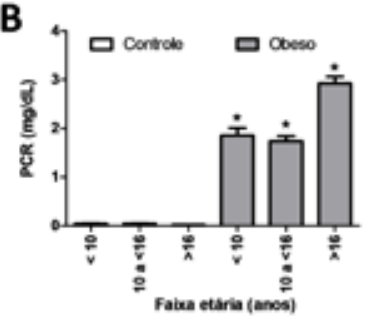

。

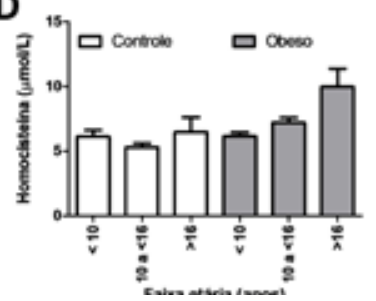

Figura 2. Representação do comportamento da proteína C reativa (PCR- us) em comparação com as variáveis HDL (A), triglicérides (B), IMC (C) e CA (D) nos grupos obeso e controle $\left(n=342,{ }^{* * *} p<0,0001\right)$.
A

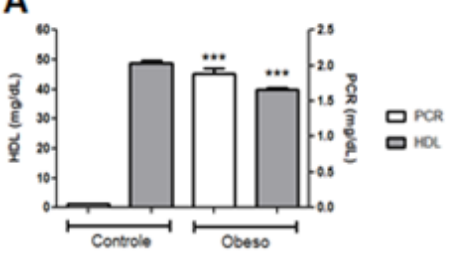

C

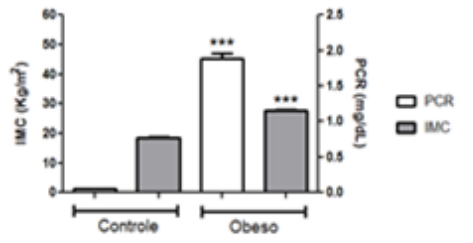

B

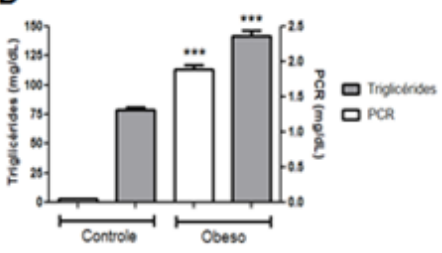

D

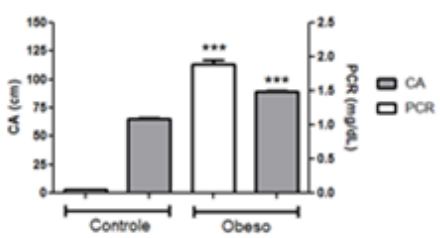

ferença estatística nos níveis da homocisteína entre os grupos obeso e controle.

Uma comparação entre as variáveis antropométricas, IMC e CA e variáveis laboratoriais, triglicérides e $\mathrm{HDL}$, de ambos os grupos da amostra está exposta na Figura 2. A representação demonstra diferenças significantes dos valores da PCR-us no grupo obeso com redução do HDL $(p=0,001)$, com elevação de triglicérides $(p=0,001)$, maior grau de IMC $(\mathrm{p}=0,001)$ e elevação da CA $(p=0,001)$.

\section{DISCUSSÃO}

Um biomarcador é um indicador de um processo biológico normal, de um processo patogênico ou de uma resposta farmacológica a um tratamento ${ }^{13}$, sendo uma medida quantificável de homeostase biológica que define o que é normal e fornece subsídios para predizer ou detectar alterações. É usado para identificar pacientes com risco aumentado de desfechos desfavoráveis segundo Marshall et a ${ }^{13}$. Ao tratar de obesidade na faixa etária pediátrica, não há consenso na literatura mundial em relação à presença de um biomarcador de comorbidades cardiometabólicas, apesar de, na população adulta, essa correlação já estar fundamentada ${ }^{14}$. Porém, estudos demonstraram que a PCR-us já apresenta níveis elevados em crianças e adolescentes com obesidade ${ }^{15,16,17}$. Isso evidencia a relação entre obesidade e início do processo inflamatório na população pediátrica obesa em relação às com peso normal ${ }^{15,16,17}$. Os resultados deste estudo corroboram esses achados, entretanto, esse aumento foi mais expressivo em ambos os sexos nos indivíduos com obesidade na faixa etária acima de 16 anos quando comparados aos controles não obesos, como demonstrado na figura 1. Esse fato se deve, provavelmente, ao maior grau de obesidade e, consequentemente, ao maior IMC, uma vez que a literatura relaciona a elevação da proteína $\mathrm{C}$ reativa sendo diretamente proporcional ao grau de $\mathrm{IMC}^{18}$. Em estudo conduzido por Kitsios et al ${ }^{19}$, os níveis de PCR-us foram significativamente maiores nos grupos obeso e com excesso de peso 
em comparação ao grupo controle. Suhet et $\mathrm{al}^{20}$, ao avaliarem 350 crianças, relataram associação significativa entre a PCR-us com excesso de peso, acreditando-se que o aumento da PCR-us possa ser um potencial mediador entre a obesidade e o início da aterosclerose na infância. Um fato relevante do nosso estudo foi a utilização da amostra pareando os sexos com a idade cronológica de acordo com a Federação Internacional de Diabetes. Isso possibilitou maior clareza da importância deste biomarcador na obesidade, mesmo em grupo pediátrico.

A PCR-us é um biomarcador inflamatório validado como preditor de risco cardiovascular em indivíduos adultos aparentemente saudáveis. O Centro de Controle de Doença Crônica (CDC) e a Sociedade Americana de Cardiologia (AHA) classificam, em relação à categoria de risco relativo cardiometabólico, os seguintes níveis da PCR-us em: risco cardíaco baixo $(<0,1 \mathrm{mg} / \mathrm{dL})$, risco cardíaco médio $(0,1-$ $0,3 \mathrm{mg} / \mathrm{dL})$ e risco cardíaco elevado $(>0,3$ $\mathrm{mg} / \mathrm{dL}$ ). Esses valores foram considerados para a população americana e europeia ${ }^{21}$. Utilizando esses critérios neste estudo, constata-se que as crianças e adolescentes com obesidade apresentaram PCR-us elevada em todas as faixas etárias. Esse resultado possibilitou a adoção de medidas dietéticas e comportamentais para uma mudança de vida dos indivíduos obesos ${ }^{30}$. Para tanto, foi necessário o esforço de uma equipe multiprofissional composta de endocrinologista, educador físico, nutricionista e psicólogo que estimularam a perda de peso das crianças e mudanças de hábitos de toda a família ${ }^{30}$. Essa proposta também foi adotada por Isasi et al ${ }^{22}$.

Com relação à homocisteína, este biomarcador cardiometabólico não demonstrou ter uma boa correlação com a obesidade na amostra avaliada. Esse fato corrobora o descrito por Anderson et al ${ }^{23}$, que observaram elevação da homocisteína em pacientes com obesidade, porém, na fase adulta. Outro estudo mostrou a associação entre homocisteína e a obesidade, mas concluiu que a homocisteína é um biomarcador cardiometabólico com baixa sensibilidade ${ }^{24}$. Atualmente, existem poucos relatos na literatura mostrando a importância deste biomarcador como ferramenta para o prognóstico de risco cardiometabólico na obesidade.

\section{A PCR-us é um}

\section{biomarcador} inflamatório validado como preditor de risco cardiovascular em indivíduos adultos

\section{aparentemente saudáveis.}

Em relação ao perfil lipídico, observou-se valores estatísticos significativos mais elevados do grupo obeso quando comparadas aos controles, com elevação de colesterol total, do LDL-c, dos triglicerídeos e redução de HDL. Esse fato é de suma importância para o viés metabólico, pois essas crianças e adolescentes com esse perfil lipídico já podem apresentar estrias de placas de gorduras a nível coronariano e carotídeo, associado à elevação da PCR-us ${ }^{25}$. Suhet et al ${ }^{20}$ observaram associação entre PCR-us e HDL baixo. Em pesquisa realizada com 124 crianças, Muramoto et al ${ }^{26}$ relataram que, para cada aumento de $1 \mathrm{mg} / \mathrm{L}$ na concentração sérica de PCR, houve uma redução de $0,072 \mathrm{mg} / \mathrm{dL}$ do $\mathrm{HDL}$, porém quando o LDL se eleva, ele infiltra o endotélio arterial, produzindo estrias gordurosas precocemente- em torno da primeira e segunda década de vida. A progressão desta dislipidemia contribui para que vários subgrupos de células brancas infiltrem a parede vascular e secretem citocinas inflamatórias, moléculas oxidativas e resultem no estado inflamatório. Essa dislipidemia mista, com posterior placa ateromatosa, foi evidenciada em crianças mexicanas descrito por Enriquez et $\mathrm{al}^{27}$.

De acordo com Weiss et $\mathrm{al}^{28}$, há uma associação positiva entre a incidência da obesidade e dislipidemia em crianças e adolescentes. Encontrou-se prevalência de cerca de 50\% de dislipidemia em crianças com IMC elevado, associado a uma maior circunferência abdominal, com posterior acúmulo de gordura visceral e esteatose hepática não alcoólica. Diante disso, o excesso de peso é considerado um critério para triagem de perfil lipídico em crianças e adolescentes obesas, conforme orientação da Sociedade Brasileira de Pediatria ${ }^{29}$. Por outro lado, a dislipidemia na infância pode estar associada ao desenvolvimento precoce de risco cardiometabólico com elevação da PCR-us, como observado em outras populações de crianças e adolescentes com obesidade e também na nossa população do estudo ${ }^{28}$.

Portanto, podemos concluir que proteína $C$ reativa ultrassensível pode ser utilizada precocemente como um importante biomarcador de risco cardiometabólico mesmo em grupos pediátricos com obesidade.

\section{REFERÊNCIAS}

1. Gupta N, Goel K, Shah P, Misra A. Childhood obesity in developing countries: Epidemiology, determinants, and prevention. Endocr Rev. 2012;33:48-70.

2. Fernandez RJ, Klimentidis YC, Keita AD, Casazza K. Genetic in- fluences in childhood obesity: recent progress and recommendations for experimental designs. International Journal of Obesity. 2012;36:479-84.

3.Ezzati M. Worldwide trends in body-mass index, underweight, 


\section{REFERÊNCIAS}

overweight, and obesity from 1975 to 2016: a pooled analysis of 2416 population-based measurement studies in 128.9 million children, adolescents, and adults. Lancet. 2017;390:2627-42.

4.Wabitsch M, Moss A, Kromeyer-Hauschild K. Unexpected plateauing of childhood obesity rates in developed countries. BMC Medicine 2014;12:1-5.

5.Rokholm B, Baker JL, Sørensen TIA. The levelling off of the obesity epidemic since the year 1999-a review of evidence and perspectives. ObesRev 2010;1:835-46.

6. IBGE. Pesquisa de Orçamentos Familiares - POF 2008-2009: http://www.ibge.gov.br/home/estatistica/populacao/condicaodevida/pof/2008_2009_encaa/comentario.pdf. Acessado em 16/11/2010.

7. Melo ME. Os Números da Obesidade no Brasil: VIGITEL 2009 e POF 2008-2009.

8. Wang Z, Nakayama T. Inflammation, a link between obesity and cardiovascular disease. Mediators of Inflammation. 2010;173-86.

9. Junior AJS. Adipocinas: a relação endócrina entre obesidade e diabetes tipo II. Revista Brasileira de Obesidade, Nutrição e Emagrecimento. 2017;11:135-144.

10. Teixeira BC, Lopes AL, Macedo RCO, Correa CS, Ramis TR, Ribeiro JL, et al. Marcadores inflamatórios, função endotelial e riscos cardiovasculares. J Vasc Bras. 2014; 13:108-115.

11.Menezes CA, Andrade LJ de O, Pinheiro JJ, Correia GS, Melo PRS de, Rios-Santos F. Polimorfismo do promotor -308 do fator de necrose tumoral alfa e resistência à insulina em adolescentes com sobrepeso e obesidade. Medicina (Ribeirão Preto). 2018; 50 (6):358-64. Disponivel em: https://www.revistas.usp.br/rmrp/ article/view/146412.

12.Aday AW and Ridker PM. Targeting Residual Inflammatory Risk: A Shifting Paradigm for Atherosclerotic Disease. Front. Cardiovasc. Med.2019; 6:16. Doi: 10.3389/fcvm.2019.00016

13. Marshall JC, Reinhart K. Biomarkers of sepsis. Crit Care Med. 2009;37: 2290-9.

14. Han TS, Sattar N, Williams K, González, VC, Lean ME, Haffner $\mathrm{SM}$. Prospective study of C-reactive protein in relation to the development of diabetes and metabolic syndrome in the Mexico City Diabetes Study. Diabetes Care. 2002;25:2016-21.

15.Guran O, Akalin F, Ayabakan C, Dereli FY, Haklar G. High- sensitivity C-reactive protein in children at risk for coronary artery disease. Acta Paediatrica. 2007; 96:1214-9.

16.Retnakaran R, Hanley AJG, Connelly PW, Harris SB, Zinman B. Elevated C-reactive protein in Native Canadian children: an ominous early complication of childhood obesity. Diabetes, Obesity and Metabolism. 2006;8:483-91.

17. Silva, LR, Stefanello JMF, Pizzi J, Timossi LS, Leite N. Aterosclerose subclínica e marcadores inflamatórios em crianças e adolescentes obesos e não obesos. Rev Bras Epidemiol 2012; 15(4): 804-16.
18. Roberts WL, Sedrick R, Moulton L, Spencer A, Rifoi N. Evaluation of four automated High sensitivity C-reactive protein methods: Implications for Clinical and Epidemiological Applications. Clinical Chemistry. 2000;46:461-6.

19.Kitsios K, Papadopoulou M, Kosta K, Kadoglou N, Papaggiani M, Tsiroukidou K. High-sensitivy c-reative protein levels and metabolic disorders in obese and overweight children and adolescentes. J Clin Res Pediatr Endocrinol 2013;5(1):44-49. DOI: 10.4274/Jcrpe.789

20.Suhet LA, Hermsdorff HHM, Rocha NP, Silva MA, Filgueiras MS, Milagres LC, Peluzio, MCG, et al. Increased C-Reactive Protein in Brazilian Children: Association with Cardiometabolic Risk and Metabolic Syndrome Components (PASE Study). Cardiology Research and Practice. 2019;1-10.

21.Khera A, McGuire DK, Murphy SA, Stanek HG, Das SR, Vongpatanasin W, Wians FH Grundy SM, Lemos JA. Race and gender differences in C-reactive protein levels. J Am Coll Cardiol. 2005; 46:464-9.

22.Isasi CR, Deckelbaum RJ, Tracy RP, Starc TJ, Berglund L, Shea $\mathrm{S}$. Physical fitness and $\mathrm{C}$ reactive protein level in children and young adults: The Columbia University BioMarkers study. Pediatrics. 2003;111:332-8.

23. Anderson JL, Muhlesteim JB, Horne BC, Carlquist JF, Madsen TE et al. Plasma homocysteine predicts mortality independently of traditional risck factors and C-reactive protein in patients with angiographically defined coronary artery disease. Circulation. 2000;102:1227-32.

24.Frauca JR, Gil EMG, LozaroGB, Etayo PM, Martínez PV, López JPR, Lozano OB, Moreno LA. Abdominal fat and metabolic risk in obese children and adolescents. J Physiol Biochem. 2009; 65:415-20.

25.Ridker, P.M. High- sensitivity C- reactive protein and cardiovascular risk: rationale for screening and primary prevention. Am J Cardiol. 2003; 92:17-22.

26.Muramoto G, Figueiredo Delgado A, Souza EC, A. Gilio E, Carvalho WB, Maranhão RC, Lipid profiles of children and adolescents with inflammatory response in a paediatric emergency department, Annals of Medicine. 2016;48:323-329.

27.Enríquez GVG, Benítez MIR, Gallegos VG, Buen EP, Sanromán RT, Cortés CLG. Contribution of TNF -308A and CCL2-2518A to carotid intima- media thickness in obese mexican children and adolescents. Archives of Med Research. 2008;39:753-9.

28. Weiss R, Dziura J, Burgert TS, Tamborlane WV, Taksali SE, Yeckel CW, et al. Obesity and the metabolic syndrome in children and adolescents. N. Engl J Med. 2004;350:2362-74.

29. Giuliano ICB, Caramelli B. Dislipidemias na infância e na adolescência. Pediatria. 2008;29:275-85.

30. Menezes CA, Loureiro ZJP, Vasconcelos, RS. Clinical, dietary and psychosocial effectiveness of interdisciplinary care in child and juvenile obesity. Journal of Pediatrics and Infants 2020; 3:2 7-12. 